\title{
First Synthesis of 4'-Selenonucleosides Showing Unusual Southern Conformation
}

\author{
Lak Shin Jeong, ${ }^{*}, 1$ Dilip K. Tosh, ${ }^{1}$ Hea Ok Kim, ${ }^{1}$ Ting Wang, ${ }^{1}$ Xiyan Hou, ${ }^{1}$ Ho Seop \\ Yun, ${ }^{2}$ Youngjoo Kwon, ${ }^{1}$ Sang Kook Lee, ${ }^{1}$ Jungwon Choi, ${ }^{\text {, }}$ and Long Xuan Zhao ${ }^{1}$ \\ ${ }^{1}$ Laboratory of Medicinal Chemistry, College of Pharmacy, Ewha Womans University, \\ Seoul 120-750, Korea, ${ }^{2}$ Division of Energy Systems Research and Department of \\ Chemistry, Ajou University, Suwon 443-749, Korea, and ${ }^{3}$ Department of Chemistry, \\ The University of Suwon, Kyunggi 445-743, Korea
}

\section{Supporting Information}

Table of Contents

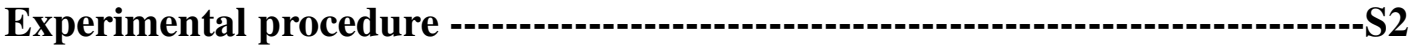

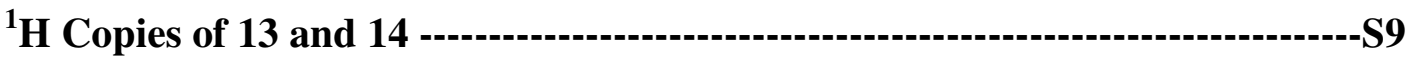

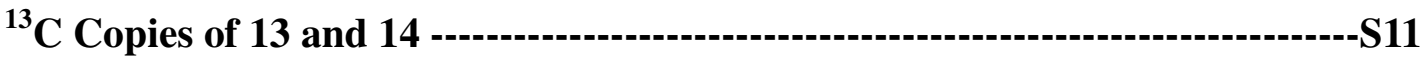




\section{General}

${ }^{1} \mathrm{H}$ NMR Spectra $\left(\mathrm{CDCl}_{3}\right.$ or $\left.\mathrm{CD}_{3} \mathrm{OD}\right)$ were recorded on Varian Unity Invoa $400 \mathrm{MHz}$. The ${ }^{1} \mathrm{H}$ NMR data are reported as peak multiplicities: $s$ for singlet, $\mathrm{d}$ for doublet, $\mathrm{dd}$ for doublet of doublets, $\mathrm{t}$ for triplet, $\mathrm{q}$ for quartet, br $\mathrm{s}$ for broad singlet and $\mathrm{m}$ for multiplet. Coupling constants are reported in hertz. ${ }^{13} \mathrm{C} \mathrm{NMR}\left(\mathrm{CDCl}_{3}\right.$ or $\left.\mathrm{CD}_{3} \mathrm{OD}\right)$ were recorded on Varian Unity Inova $100 \mathrm{MHz}$. The chemical shifts were reported as parts per million $(\delta)$ relative to the solvent peak. Optical rotations were determined on Jasco in methanol. UV spectra were recorded on U-3000 made by Histachi in methanol. FAB mass spectra were recorded on Jeol HX 110 spectrometer. Elementary analyses were measured on EA1110. Melting points were measured on B-540 made by Buchi. Reactions were checked with TLC (Merck precoated 60F254 plates). Spots were detected by viewing under a UV light, colorizing with charring after dipping in anisaldehyde solution with acetic acid, sulfuric acid and methanol. Column chromatography were performed on silica gel 60 (230-400 mesh, Merck). Reagents were purchased from Aldrich Chemical Company. Solvents were obtained from local suppliers. All the anhydrous solvents were distilled over $\mathrm{CaH}_{2}, \mathrm{P}_{2} \mathrm{O}_{5}$ or sodium/benzophenone prior to the reaction.

\section{2,3;5,6-Di- $O$-Isopropylidene-D-gulonic y-lactol (2).}

To a solution of $\mathbf{1}(5.00 \mathrm{~g}, 19.4 \mathrm{mmol})$ in THF ( $80 \mathrm{~mL})$ was added DIBAL (19.3 mL. 1.0 M solution in THF, $19.3 \mathrm{mmol}$ ) at $-78{ }^{\circ} \mathrm{C}$ and the reaction was stirred at the same temperature for $0.5 \mathrm{~h}$ and quenched by slow addition of methanol. The colloidal suspension was filtered through a Celite pad and partitioned between ethyl acetate and water. The combined organic layers were dried $\left(\mathrm{MgSO}_{4}\right)$, filtered, and evaporated. The residue was purified on flash silica gel column chromatography (hexane:ethyl acetate = 2:1) to give the lactol $2(4.14 \mathrm{~g}, 82 \%)$ as a white solid: Data for major anomer: mp 151$152{ }^{\circ} \mathrm{C}$; MS (FAB) m/z $261\left(\mathrm{M}+\mathrm{H}^{+}\right) ;[\alpha]^{20}{ }_{\mathrm{D}}-51.80\left(c \quad 0.16, \mathrm{CH}_{3} \mathrm{OH}\right) ;{ }^{1} \mathrm{H}$ NMR $\left(\mathrm{CD}_{3} \mathrm{OD}\right) \delta 1.03(\mathrm{~s}, 3 \mathrm{H}), 1.05(\mathrm{~s}, 3 \mathrm{H}), 1.08(\mathrm{~s}, 3 \mathrm{H}), 1.1$ (s, 3H), $3.46(\mathrm{dd}, 1 \mathrm{H}, J=7.0$, $8.4 \mathrm{~Hz}), 3.83-3.88(\mathrm{~m}, 1 \mathrm{H}), 3.94-3.98(\mathrm{~m}, 1 \mathrm{H}), 4.22$ (d, $J=6.0 \mathrm{~Hz}, 1 \mathrm{H}), 4.44-4.46$ (m, $1 \mathrm{H}), 4.52-4.62(\mathrm{~m}, 2 \mathrm{H}) ;{ }^{13} \mathrm{C}$ NMR $\left(\mathrm{CD}_{3} \mathrm{OD}\right) \delta 24.9,25.9,26.4,27.1,67.1,77.3,81.4$, 83.3, 87.3, 102.5, 110.8, 113.9; Anal. Calcd for $\mathrm{C}_{12} \mathrm{H}_{20} \mathrm{O}_{6}$ : C, 55.37; H, 7.74. Found: $\mathrm{C}$, $55.86 ; \mathrm{H}, 7.88$.

\section{2,3-O-Isopropylidene-D-gulonic 8 -lactol (3).}

A solution of 2 (4.14 g, $18.79 \mathrm{mmol})$ with $80 \%$ acetic acid was stirred at room temperature for $5 \mathrm{~h}$. The solvent was evaporated and the residue was purified on flash silica gel column chromatography (methylene chloride:methanol $=10: 1)$ to give $\mathbf{3}(2.81$ 
g, 81\%) as a white solid: Data for major anomer: mp 140-145 ${ }^{\circ} \mathrm{C}$; MS (FAB) m/z 221 $\left(\mathrm{M}+\mathrm{H}^{+}\right) ;[\alpha]^{20}{ }_{\mathrm{D}}-63.04 ;\left(c 0.13, \mathrm{CH}_{3} \mathrm{OH}\right) ;{ }^{1} \mathrm{H} \mathrm{NMR}\left(\mathrm{CD}_{3} \mathrm{OD}\right) \delta 1.29(\mathrm{~s}, 3 \mathrm{H}), 1.41$ (s, $3 \mathrm{H}), 3.61-3.65(\mathrm{~m}, 1 \mathrm{H}), 3.72-3.76(\mathrm{~m}, 1 \mathrm{H}), 3.88-3.93(\mathrm{~m}, 1 \mathrm{H}), 4.07-4.10(\mathrm{~m}, 1 \mathrm{H}), 4.5(\mathrm{~d}$, $1 \mathrm{H}, J=5.6 \mathrm{~Hz}), 4.76-4.78(\mathrm{~m}, 1 \mathrm{H}), 5.24(\mathrm{~s}, 1 \mathrm{H}) ;{ }^{13} \mathrm{C} \mathrm{NMR}\left(\mathrm{CD}_{3} \mathrm{OD}\right) \delta 24.9,26.5,64.3$, 72.8, 81.4, 81.6, 87.8, 102.0, 113.6; Anal. Calcd for $\mathrm{C}_{9} \mathrm{H}_{16} \mathrm{O}_{6}: \mathrm{C}, 49.09$; H, 7.32. Found C, 49.34; H, 7.30.

\section{2,3-O-Isopropylidene-L-lyxose (4).}

To a solution of diol 3 (2.0 g, $9.0 \mathrm{mmol})$ in $\mathrm{EtOH}(50 \mathrm{~mL})$ was added aqueous solution of sodium metaperiodate $(2.89 \mathrm{~g}, 13.5 \mathrm{mmol})$ at $0{ }^{\circ} \mathrm{C}$. After starting material disappeared, sodium borohydride $(0.37 \mathrm{~g}, 9.9 \mathrm{mmol})$ was added to the reaction mixture at $0{ }^{\circ} \mathrm{C}$ and the mixture was stirred at the same temperature until the aldehyde was consumed. The reaction mixture was filtered through a Celite pad and the filtrate was evaporated. The residue was partitioned between ethyl acetate and water. The organic layer was dried $\left(\mathrm{MgSO}_{4}\right)$, filtered, and evaporated. The residue was purified by flash silica gel column chromatography (methylene chloride:methanol $=10: 1)$ to give $4(1.15$ $\mathrm{g}, 67 \%$ ) whose spectral data were identical with those of authentic sample (Ref: Varela, O.; Zunszain, P. A. J. Org. Chem. 1993, 58, 7860-7864; Xie, M.; Berges, D. A.; Robins, M. J. J. Org. Chem. 1996, 61, 5178-5179)

\section{5-tert-Butyldiphenylsilyl-2,3-O-isopropylidene-L-lyxose (5).}

To a solution of 4 (4.05 g, $21.29 \mathrm{mmol})$, in methylene chloride (100 mL) were added triethylamine $(5.92 \mathrm{~mL}, 42.49 \mathrm{mmol})$, dimethylaminopyridine $(0.52 \mathrm{~g}, 4.25 \mathrm{mmol})$, and TBDPSCl $(8.77 \mathrm{~mL}, 31.9 \mathrm{mmol})$ and the mixture was stirred at room temperature for $3.5 \mathrm{~h}$. The solvent was evaporated and the residue was partitioned between methylene chloride and water. The organic layer was dried $\left(\mathrm{MgSO}_{4}\right)$, filtered and evaporated. The residue was purified on flash silica gel column chromatography (hexane:ethyl acetate = 10:1) to give $5(8.4 \mathrm{~g}, 92 \%)$ as a colorless syrup: Data for major anomer: MS (FAB) $\mathrm{m} / \mathrm{z}$ $429\left(\mathrm{M}+\mathrm{H}^{+}\right) ;[\alpha]^{20}{ }_{\mathrm{D}} 2.52\left(c 0.19, \mathrm{CH}_{3} \mathrm{OH}\right) ;{ }^{1} \mathrm{H}$ NMR $\left(\mathrm{CDCl}_{3}\right) \delta 1.06(\mathrm{~s}, 9 \mathrm{H}), 1.28(\mathrm{~s}$, $3 \mathrm{H}), 1.35(\mathrm{~s}, 3 \mathrm{H}), 3.88-3.93(\mathrm{~m}, 1 \mathrm{H}), 3.96-4.01(\mathrm{~m}, 1 \mathrm{H}), 4.31-4.35(\mathrm{~m}, 1 \mathrm{H}), 4.58(\mathrm{~d}, 1 \mathrm{H}$, $J=6.0 \mathrm{~Hz}), 4.72-4.76(\mathrm{~m}, 1 \mathrm{H}), 5.37(\mathrm{~s}, 1 \mathrm{H}), 7.35-7.44(\mathrm{~m}, 6 \mathrm{H}), 7.67-7.73(\mathrm{~m}, 4 \mathrm{H}) ;{ }^{13} \mathrm{C}$ NMR $\left(\mathrm{CDCl}_{3}\right) \delta 19.4,25.1,25.2,26.0,26.2,27.0,61.5,62.2,76.4,78.7,79.5,80.0$, 81.1, 85.6, 97.0, 101.5, 112.6, 127.7, 127.8, 129.8, 133.7, 135.8, 135.9; Anal. Calcd for $\mathrm{C}_{24} \mathrm{H}_{32} \mathrm{O}_{5} \mathrm{Si}: \mathrm{C}, 67.26 ; \mathrm{H}, 7.53$. Found $\mathrm{C}, 66.86 ; \mathrm{H}, 7.13$.

\section{5-O-tert-Butyldiphenylsilyl-2,3-O-isopropylidene-L-lyxitol (6).}


To a stirred solution of 5 (16. $1 \mathrm{~g}, 37.56 \mathrm{mmol})$ in methanol $(350 \mathrm{~mL})$ was added sodium borohydride $(4.26 \mathrm{~g}, 112.6 \mathrm{mmol})$ at $0^{\circ} \mathrm{C}$ and the mixture was stirred at room temperature for $2 \mathrm{~h}$. After the solvent was evaporated, the residue was partitioned between ethyl acetate and water. The organic layer was dried $\left(\mathrm{MgSO}_{4}\right)$, filtered and evaporated. The residue was purified on flash silica gel column chromatography (hexane: ethyl acetate $=2: 1)$ to give $6(15.8 \mathrm{~g}, 98 \%)$ as a colorless syrup: MS (FAB) $\mathrm{m} / \mathrm{z} 453\left(\mathrm{M}+\mathrm{Na}^{+}\right) ;[\alpha]^{20}{ }_{\mathrm{D}} 6.66 ;\left(c 0.28, \mathrm{CH}_{3} \mathrm{OH}\right) ;{ }^{1} \mathrm{H}$ NMR $\left(\mathrm{CDCl}_{3}\right) \delta 1.08(\mathrm{~s}, 9 \mathrm{H}), 1.28$ $(\mathrm{s}, 3 \mathrm{H}), 1.56(\mathrm{~s}, 3 \mathrm{H}), 3.93-4.04(\mathrm{~m}, 3 \mathrm{H}), 4.68(\mathrm{dd}, 1 \mathrm{H}, J=3.6,4.0 \mathrm{~Hz}), 4.74(\mathrm{dd}, 1 \mathrm{H}, J=$ 3.6, $4.0 \mathrm{~Hz}), 5.57(\mathrm{dd}, 1 \mathrm{H}, J=2.0,8.0 \mathrm{~Hz}), 6.35$ (d, 1H, $J=4.0 \mathrm{~Hz}), 7.37-7.47$ (m, 6H), $7.51(\mathrm{~d}, 1 \mathrm{H}, J=8.0 \mathrm{~Hz}), 7.64-7.69(\mathrm{~m}, 4 \mathrm{H}), 8.77$ (br s, $1 \mathrm{H}) ;{ }^{13} \mathrm{C} \mathrm{NMR}\left(\mathrm{CDCl}_{3}\right) \delta 19.5$, 25.5, 27.1, 28.0, 50.4, 59.7, 66.1, 85.3, 90.1, 103.5, 112.4, 128.0, 128.1, 130.3, 130.4, 132.8, 133.2, 135.7, 1358, 142.0, 150.0, 162.8; Anal. Calcd. for $\mathrm{C}_{24} \mathrm{H}_{34} \mathrm{O}_{5} \mathrm{Si}: \mathrm{C}, 66.94$; H, 7.96. Found C, 67.30; H, 8.00.

\section{5-O-tert-Butyldiphenylsilyl-2,3-O-isopropylidene-1,4-di- $O$-methanesulfonyl-L- lyxitol (7).}

To a solution of $6(1.05 \mathrm{~g}, 2.43 \mathrm{mmol})$ and DMAP $(0.006 \mathrm{~g}, 0.049 \mathrm{mmol})$ in methylene chloride $(32 \mathrm{~mL})$ were added triethylamine $(2.72 \mathrm{~mL}, 19.46 \mathrm{mmol})$ and mesyl chloride $(0.75 \mathrm{~mL}, 9.7 \mathrm{mmol})$ at $0{ }^{\circ} \mathrm{C}$ and the reaction mixture was stirred at room temperature for $1 \mathrm{~h}$. Saturated aqueous solution of $\mathrm{NaHCO}_{3}$ was added to the mixture and the aqueous layer was extracted with methylene chloride. The organic layer was dried $\left(\mathrm{MgSO}_{4}\right)$, filtered, and evaporated. The residue was purified on flash silica gel column chromatography (hexane:ethyl acetate $=2: 1)$ to give $7(1.39 \mathrm{~g}, 97 \%)$ as a colorless syrup: MS (FAB) m/z $609\left(\mathrm{M}+\mathrm{Na}^{+}\right) ;[\alpha]^{20}{ }_{\mathrm{D}}-3.30\left(c 0.21, \mathrm{CH}_{3} \mathrm{OH}\right) ;{ }^{1} \mathrm{H}$ NMR $\left(\mathrm{CDCl}_{3}\right) \delta$ 1.09 (s, 9H), 1.37 (s, 3H), 1.49 (s, 3H), 3.00 (s, 3H), 3.07 (s, 3H), 3.92 (dd, 1H, J = 5.2, $11.0 \mathrm{~Hz}), 3.95(\mathrm{dd}, 1 \mathrm{H}, J=5.2,11.0 \mathrm{~Hz}), 4.27-4.33(\mathrm{~m}, 2 \mathrm{H}), 4.36-4.39(\mathrm{~m}, 1 \mathrm{H}), 4.53(\mathrm{t}$, $1 \mathrm{H}, J=6.2 \mathrm{~Hz}), 4.82(\mathrm{q}, 1 \mathrm{H}, J=5.2 \mathrm{~Hz}), 7.40-7.46(\mathrm{~m}, 6 \mathrm{H}), 7.66-7.70(\mathrm{~m}, 4 \mathrm{H}) ;{ }^{13} \mathrm{C}$ NMR $\left(\mathrm{CDCl}_{3}\right) \delta 19.2,25.4,26.8,27.2,37.5,39.0,63.6,67.6,74.3,75.0,79.1,109.6$, $128.1,128.2$, 130.2, 132.2, 132.3, 135.5, 135.6; Anal. Calcd for $\mathrm{C}_{26} \mathrm{H}_{38} \mathrm{O}_{9} \mathrm{~S}_{2} \mathrm{Si}: \mathrm{C}$, 53.22; H, 6.53. Found C, 53.11; H, 6.33.

\section{1,4-Anhydro-5-O-tert-butyldiphenylsilyl-2,3-O-isopropylidene-4-seleno-D-ribitol (8).}

To a suspension of selenium powder $(1.08 \mathrm{~g}, 13.67 \mathrm{mmol})$ in ethanol $(90 \mathrm{~mL})$ was added sodium borohydride at room temperature until the color of the reaction mixture changed from black to colorless. Compound 7 (3.93 g, $6.69 \mathrm{mmol})$ in THF (50 mL) was then added to the mixture and heated at $60{ }^{\circ} \mathrm{C}$ overnight. The solvent was evaporated, 
the residue was dissolved in ethyl acetate $(50 \mathrm{~mL})$, and the mixture was washed with water $(3 \times 30 \mathrm{~mL})$, brine, dried $\left(\mathrm{MgSO}_{4}\right)$, filtered, and evaporated. The residue was purified on flash silica gel column chromatography (hexane: ethyl acetate $=10: 1$ ) to give the selenosugar $8(3.0 \mathrm{~g}, 96 \%)$ as pale yellow syrup: MS (FAB) $\mathrm{m} / \mathrm{z} 475\left(\mathrm{M}-\mathrm{H}^{+}\right) ;[\alpha]^{20}{ }_{\mathrm{D}}$ $54.30\left(c \mathrm{0} 0.15, \mathrm{CH}_{3} \mathrm{OH}\right) ;{ }^{1} \mathrm{H} \mathrm{NMR}\left(\mathrm{CDCl}_{3}\right) \delta 1.07(\mathrm{~s}, 9 \mathrm{H}), 1.31(\mathrm{~s}, 3 \mathrm{H}), 1.52(\mathrm{~s}, 3 \mathrm{H})$, $2.96(\mathrm{dd}, 1 \mathrm{H}, J=2.0,11.4 \mathrm{~Hz}), 3.14(\mathrm{dd}, 1 \mathrm{H}, J=5.2,11.4 \mathrm{~Hz}), 3.63-3.67$ (m, 1H), 3.70$3.74(\mathrm{~m}, 1 \mathrm{H}), 3.84-3.88(\mathrm{~m}, 1 \mathrm{H}), 4.78(\mathrm{dd}, 1 \mathrm{H}, J=1.8,5.2 \mathrm{~Hz}), 4.82-4.85(\mathrm{~m}, 1 \mathrm{H})$, 7.26-7.46 (m, 6H), 7.65-7.70 (m, 4H); ${ }^{13} \mathrm{C} \mathrm{NMR}\left(\mathrm{CDCl}_{3}\right) \delta$ 19.4, 24.8, 27.0, 27.1, 30.0, 50.4, 66.6, 85.3, 87.5, 110.5, 127.9, 130.0, 130.1, 133.3, 133.4, 135.8; Anal. Calcd for $\mathrm{C}_{24} \mathrm{H}_{32} \mathrm{O}_{3} \mathrm{SeSi}$ : C, 60.61; H, 6.78. Found C, 60.98; H, 7.13.

\section{1-(5-O-tert-Butyldiphenylsilyl-2,3-O-isopropylidene-4-seleno- $\beta$-D- ribofuranosyl)uracil (11).}

To a stirred solution of seleno sugar $8(3.50 \mathrm{~g}, 7.36 \mathrm{mmol})$ in $\mathrm{CH}_{2} \mathrm{Cl}_{2}(60 \mathrm{~mL})$ was added a solution of $m$-CPBA $(1.81 \mathrm{~g}, 7.35 \mathrm{mmol}, 70 \%)$ in $\mathrm{CH}_{2} \mathrm{Cl}_{2}(25 \mathrm{~mL})$ at $-78{ }^{\circ} \mathrm{C}$ and the mixture was stirred at the same temperature for $45 \mathrm{~min}$. The reaction mixture was quenched with saturated $\mathrm{NaHCO}_{3}$ solution and extracted with $\mathrm{CH}_{2} \mathrm{Cl}_{2}$. The organic layer was washed with brine, dried $\left(\mathrm{MgSO}_{4}\right)$, filtered, and evaporated. The residue was purified on flash silica gel column chromatography (methylene chloride:methanol = $30: 1)$ to give the selenoxide $9(3.0 \mathrm{~g}, 85 \%)$ as a colorless syrup. Due to unstable nature of the selenoxide 9, it was immediately used for next step.

A suspension of uracil $(1.35 \mathrm{~g}, 12.05 \mathrm{mmol})$ in toluene $(48 \mathrm{~mL})$ was treated with triethylamine $(3.4 \mathrm{~mL}, 24.39 \mathrm{mmol})$ and TMSOTf $(9.0 \mathrm{~mL}, 50.06 \mathrm{mmol})$ and the resulting mixture was stirred at room temperature for $1 \mathrm{~h}$. After adding additional methylene chloride $(24 \mathrm{~mL})$, the silylated uracil was added to a solution of 9 (3.0 g, $6.10 \mathrm{mmol}$ ) in methylene chloride $(24 \mathrm{~mL})$ slowly over a period of $30 \mathrm{~min}$ at $0{ }^{\circ} \mathrm{C}$. An additional amount of triethylamine $(3.4 \mathrm{~mL}, 24.39 \mathrm{mmol})$ in toluene was added dropwise to the reaction mixture to initiate the Pummerer reaction at $0{ }^{\circ} \mathrm{C}$. After overnight stirring at room temperature, water $(40 \mathrm{~mL})$ was added and the aqueous layer was extracted with methylene chloride $(3 \mathrm{X} 40 \mathrm{~mL}$ ). The combined organic layers were washed with saturated $\mathrm{NaHCO}_{3}$ solution $(30 \mathrm{~mL})$ and brine $(30 \mathrm{~mL})$, dried $\left(\mathrm{MgSO}_{4}\right)$, filtered, and evaporated. The residue was purified on flash silica gel column chromatography (hexane:ethylacetate $=2: 1$ ) to give the uracil condensed compound $\mathbf{1 1}$ $(1.65 \mathrm{~g}, 53 \%)$ as a light yellow foam: $\mathrm{mp} 71-73{ }^{\circ} \mathrm{C}$; UV (MeOH) $\lambda_{\max } 265 \mathrm{~nm}$; MS (FAB) $\mathrm{m} / \mathrm{z} 587\left(\mathrm{M}+\mathrm{H}^{+}\right) ;[\alpha]^{20}{ }_{\mathrm{D}}-47.74 ;\left(c\right.$ 0.22, $\left.\mathrm{CH}_{3} \mathrm{OH}\right) ;{ }^{1} \mathrm{H}$ NMR $\left(\mathrm{CDCl}_{3}\right) \delta 1.08(\mathrm{~s}$, 9H), 1.28 (s, 3H), 1.56 (s, 3H), 3.95-4.02 (m, 3H), 4.67 (dd, 1H, J = 4.2, $5.2 \mathrm{~Hz}), 4.73$ 
$(\mathrm{dd}, 1 \mathrm{H}, J=4.2,5.2 \mathrm{~Hz}), 5.56(\mathrm{dd}, 1 \mathrm{H}, J=2.0,8.0 \mathrm{~Hz}), 6.35(\mathrm{~d}, 1 \mathrm{H}, J=4.0 \mathrm{~Hz}), 7.37-$ $7.45(\mathrm{~m}, 6 \mathrm{H}), 7.52(\mathrm{~d}, 1 \mathrm{H}, J=8.4 \mathrm{~Hz}), 7.63-7.69(\mathrm{~m}, 4 \mathrm{H}), 8.45$ (br s, $1 \mathrm{H}) ;{ }^{13} \mathrm{C}$ NMR $\left(\mathrm{CDCl}_{3}\right) \delta 19.5,25.5,26.8,27.0,27.1,28.0,50.3,59.7,66.1,76.6,85.3,90.1,103.5$, 112.4, 128.1, 128.2, 130.3, 132.8, 133.2, 135.7, 135.8, 142.0, 150.0; Anal. Calcd for $\mathrm{C}_{28} \mathrm{H}_{34} \mathrm{~N}_{2} \mathrm{O}_{5} \mathrm{SeSi}$ : C, 57.43; H, 5.85; N, 4.78. Found C, 57.04; H, 5.86; N, 4.66.

\section{$N^{4}$-Benzoyl-1-(5-O-tert-Butyldiphenylsilyl-2,3-O-isopropylidene-4-seleno- $\beta$-D- ribofuranosyl)cytosine (12).}

Selenoxide $\mathbf{9}$ was converted to compound $\mathbf{1 2}$ as yellowish foam according to the same procedure used in the preparation of 11: yield 35\%; mp 91-93 ${ }^{\circ} \mathrm{C}$; $\mathrm{UV}(\mathrm{MeOH}) \lambda_{\max } 310$ $\mathrm{nm} ; \mathrm{MS}(\mathrm{FAB}) \mathrm{m} / \mathrm{z} 690\left(\mathrm{M}+\mathrm{H}^{+}\right) ;[\alpha]^{20}{ }_{\mathrm{D}}-44.12\left(c 0.55, \mathrm{CH}_{3} \mathrm{OH}\right) ;{ }^{1} \mathrm{H}$ NMR $\left(\mathrm{CDCl}_{3}\right) \delta$ 1.09 (s, 9H), 1.28 (s, 3H), 1.57 (s, 3H), 3.96-4.05 (m, 3H), $4.78(\mathrm{dd}, 1 \mathrm{H}, J=3.4,5.4 \mathrm{~Hz})$, $4.83(\mathrm{dd}, 1 \mathrm{H}, \quad J=2.8,5.4 \mathrm{~Hz}), 6.40(\mathrm{~d}, 1 \mathrm{H}, J=3.6 \mathrm{~Hz}) 7.36-7.46(\mathrm{~m}, 7 \mathrm{H}), 7.48(\mathrm{~m}$, 2H), 7.59-7.67 (m, 5H), 7.90 (d, 2H, $J=7.2 \mathrm{~Hz}), 7.96(\mathrm{~d}, 1 \mathrm{H}, J=7.2 \mathrm{~Hz}), 8.73$ (br s, $1 \mathrm{H}) ;{ }^{13} \mathrm{C} \mathrm{NMR}\left(\mathrm{CDCl}_{3}\right) \delta 19.5,25.4,27.1,27.9,51.8,66.1$ 85.9, 90.8, 112.1, 127.7, 128.1, 128.2, 129.2, 130.26, 130.24, 133.0, 133.2, 133.5, 135.7, 135.8; Anal. Calcd for $\mathrm{C}_{35} \mathrm{H}_{39} \mathrm{~N}_{3} \mathrm{O}_{5}$ SeSi: C, 61.03; H, 5.71; N, 6.10. Found: C, 60.87; H, 5.91; N, 5.70.

\section{1-(4-Seleno- $\beta$-D-ribofuranosyl)uracil (13).}

A solution of $11(0.650 \mathrm{~g}, 1.10 \mathrm{mmol})$ in $50 \%$ aqueous trifluroacetic acid $(25 \mathrm{~mL})$ was stirred at room temperature for $1 \mathrm{~h}$. The solvent was evaporated and further coevaporated with toluene to give a white solid, which was recrystallized from methanolwater (1:1) to give the final nucleoside $13(0.28 \mathrm{~g}, 81 \%)$ as a colorless solid: $\mathrm{mp} 198$ $200{ }^{\circ} \mathrm{C}$; UV $(\mathrm{MeOH}) \lambda_{\max } 267 \mathrm{~nm} ; \mathrm{MS}(\mathrm{FAB}) \mathrm{m} / \mathrm{z} 307\left(\mathrm{M}^{+}\right) ;[\alpha]_{\mathrm{D}}^{20}-113.93 ;(c 0.33$, $\left.\mathrm{CH}_{3} \mathrm{OH}\right) ;{ }^{1} \mathrm{H} \mathrm{NMR}\left(\mathrm{CD}_{3} \mathrm{OD}\right) \delta 3.40-3.44(\mathrm{~m} 1 \mathrm{H}), 3.63(\mathrm{dd}, J=7.8,11.4 \mathrm{~Hz}, 1 \mathrm{H}), 3.75$ $(\mathrm{dd}, J=7.8,11.4 \mathrm{~Hz}, 1 \mathrm{H}), 4.19(\mathrm{t}, J=2.5 \mathrm{~Hz}, 1 \mathrm{H}), 4.26(\mathrm{dd}, J=3.6,8.4 \mathrm{~Hz}, 1 \mathrm{H}), 5.77$ $(\mathrm{d}, J=8.0 \mathrm{~Hz}, 1 \mathrm{H}), 6.11(\mathrm{~d}, J=8.8 \mathrm{~Hz}, 1 \mathrm{H}), 8.0(\mathrm{~d}, J=8.0 \mathrm{~Hz}, 1 \mathrm{H}) ;{ }^{13} \mathrm{C} \mathrm{NMR}$ $\left(\mathrm{CD}_{3} \mathrm{OD}\right) \delta 48.8,55.3,63.6,73.5,77.0,102.1,142.1,150.9$, 163.0; Anal. Calcd for $\mathrm{C}_{9} \mathrm{H}_{12} \mathrm{~N}_{2} \mathrm{O}_{5} \mathrm{Se}: \mathrm{C}, 35.19 ; \mathrm{H}, 3.94 ; \mathrm{N}, 9.12$. Found C, 34.93; H, 3.82; N, 9.07.

\section{1-(4-Seleno- $\beta$-D-ribofuranosyl)cytosine (14).}

A solution of $12(0.15 \mathrm{~g}, 0.213 \mathrm{mmol})$ in $50 \%$ trifluoro acetic acid was stirred at $\mathrm{rt}$ for 1 h. Solvent was evaporated, co-evaporated with toluene and the crude residue was stirred with methanolic ammonia at $\mathrm{rt}$ for overnight. Solvent was evaporated and the residue was purified on acidic resin (DOWEX50) (ammonia: water =1:2) to give the $14(0.053$ g, 82\%) as a colorless solid: mp $150-153{ }^{\circ} \mathrm{C}$; UV (MeOH) $\lambda_{\max } 277 \mathrm{~nm}$; MS (FAB) m/z 
$307\left(\mathrm{M}^{+}\right) ;[\alpha]^{20}{ }_{\mathrm{D}}-260.0 ;\left(c\right.$ 0.17, $\left.\mathrm{CH}_{3} \mathrm{OH}\right) ;{ }^{1} \mathrm{H}$ NMR $\left(\mathrm{CD}_{3} \mathrm{OD}\right) \delta 3.56-3.63(\mathrm{~m}, 1 \mathrm{H}), 3.80$ $(\mathrm{dd}, 1 \mathrm{H}, J=5.6,11.6 \mathrm{~Hz}), 3.90(\mathrm{dd}, 1 \mathrm{H}, J=6.4,11.6 \mathrm{~Hz}), 4.21(\mathrm{t}, 1 \mathrm{H}, J=3.6 \mathrm{~Hz}) ; 4.32$ $(\mathrm{dd}, 1 \mathrm{H}, J=3.0,6.8 \mathrm{~Hz}) ; 5.95(\mathrm{~d}, 1 \mathrm{H}, J=7.6 \mathrm{~Hz}) ; 6.27(\mathrm{~d}, 1 \mathrm{H}, J=7.2 \mathrm{~Hz}) ; 8.13(\mathrm{~d}, 1 \mathrm{H}$, $J=7.6 \mathrm{~Hz}) ;{ }^{13} \mathrm{C}$ NMR $\left(\mathrm{CD}_{3} \mathrm{OD}\right) \delta 59.1,66.2,76.5,80.5,96.7,144.7,159.0,167.4$; Anal. Calcd for $\mathrm{C}_{9} \mathrm{H}_{13} \mathrm{~N}_{3} \mathrm{O}_{4}$ Se: C, 35.31; H, 4.28; N, 13.72. Found: C, 35.13; H, 3.98; N, 13.38 .

\section{NMR experiments}

All NMR experiments were performed on the JEOL ECA $600 \mathrm{MHz}$ spectrometer. Uridine or 4'-selenouridine $\left(1.0 \mathrm{mg}\right.$ ) was dissolved in $0.5 \mathrm{~mL}$ of $99.95 \% \mathrm{D}_{2} \mathrm{O}$ (Wako Co., Japan). The data were processed using Delta ${ }^{\mathrm{TM}}$ (JEOL), and proton chemical shifts were referenced to the HOD resonance (4.70 ppm at $298 \mathrm{~K}$; temperature correction factor: - $0.0109 \mathrm{ppm} / \mathrm{K})$. All one-dimensional $\left({ }^{1} \mathrm{D}\right){ }^{1} \mathrm{H}$ NMR spectra were recorded with 10 ppm spectral width, 8192 data points, and $298 \mathrm{~K}$ temperature. Suppression of the HOD resonance was achieved by a presaturation pulse. Two-dimensional (2D) experiments of COSY, TOCSY (88-ms mixing time), and NOESY (200, $500 \mathrm{~ms}$, and $1 \mathrm{~s}$ mixing times) were recorded with 4096 data points in the $t_{2}$ dimension and 512 increments. Complete assignments and coupling constants of the proton signals were achieved by a combination of NOESY, COSY, and TOCSY experiments. $90^{\circ}$-phase shifted sine bell function was used in the $t_{2}$ and $t_{1}$ dimensions prior to Fourier transformation of NOESY, while a $30^{\circ}$-phase shifted skewed sine bell function was for TOCSY and COSY. The final spectral matrices consisted of $1,024 \times 1,024$ of the ${ }^{1} \mathrm{H}$ frequency (F2 and F1) dimensions.

Table 1. ${ }^{1} \mathrm{H}$ chemical shifts of uridine and $4{ }^{\prime}$-selenouridine ${ }^{a}$

\begin{tabular}{lll}
\hline Proton & Uridine & 4'-Selenouridine \\
\hline H6 & $7.75(\mathrm{~d})^{b}$ & $8.02(\mathrm{~d})$ \\
H5 & $5.77(\mathrm{~d})$ & $5.84(\mathrm{~d})$ \\
H1' & $5.78(\mathrm{~d})$ & $6.07(\mathrm{~d})$ \\
H2' & $4.23(\mathrm{t})$ & $4.36(\mathrm{dd})$ \\
H3' & $4.10(\mathrm{t})$ & $4.22(\mathrm{t})$ \\
$\mathrm{H} 4^{\prime}$ & $4.00(\mathrm{~m})$ & $3.51(\mathrm{~m})$ \\
\hline
\end{tabular}


${ }^{a}$ Non exchangeable ${ }^{1} \mathrm{H}$ chemical shifts are mainly from the spectrum collected at $298 \mathrm{~K}$ in $\mathrm{D}_{2} \mathrm{O}$.

${ }^{b}$ The number is chemical shift as ppm and the letter in parenthesis means the peak split pattern.

${ }^{c} \mathrm{H}^{\prime}$ and $\mathrm{H} 5 "$ protons were assigned arbitrarily.

Table 2. $J$ coupling constants of uridine and $4^{\prime}$-selenouridine ${ }^{a}$

\begin{tabular}{lcc}
\hline$J$ coupling & Uridine & 4'-Selenouridine \\
\hline${ }^{3} J_{\mathrm{H} 5-\mathrm{H} 6}$ & 8.22 & 8.22 \\
${ }^{3} J_{\mathrm{H} 1^{\prime}-\mathrm{H} 2^{\prime}}$ & 4.62 & 8.70 \\
${ }^{3} J_{\mathrm{H}^{\prime}-\mathrm{H} 3^{\prime}}$ & 5.04 & 3.24 \\
${ }^{3} J_{\mathrm{H}^{\prime}-\mathrm{H} 4^{\prime}}$ & 5.52 & 3.18 \\
${ }^{3} J_{\mathrm{H}^{\prime}-\mathrm{H} 5^{\prime} / \mathrm{H}^{\prime \prime}}$ & $3.24 / 4.56$ & $5.94 / 6.84$ \\
\hline
\end{tabular}

a $J$ coupling constants as $\mathrm{Hz}$ are mainly from the spectrum collected at $298 \mathrm{~K}$ in $\mathrm{D}_{2} \mathrm{O}$. 


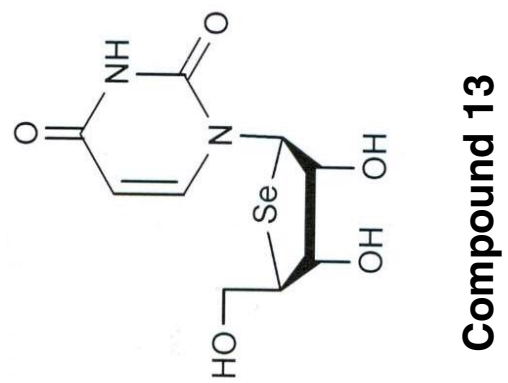

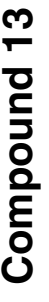

S9S. 乙
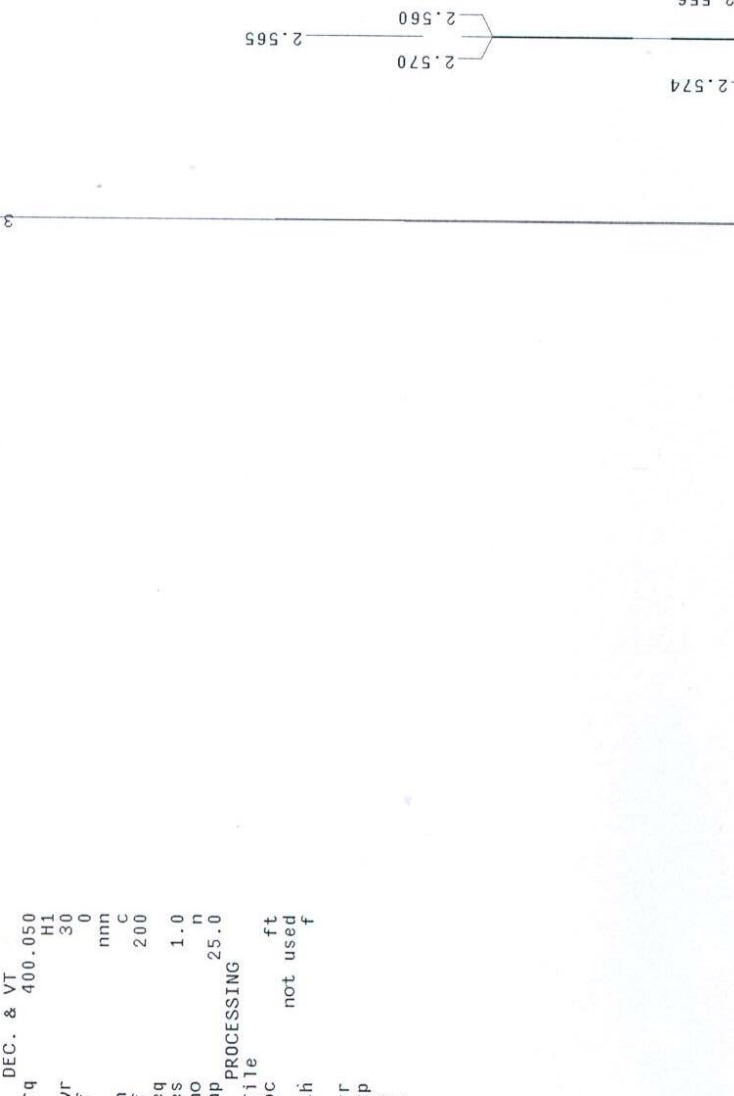

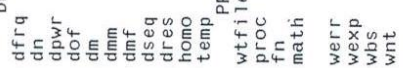

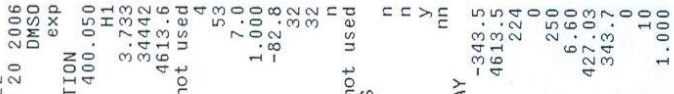
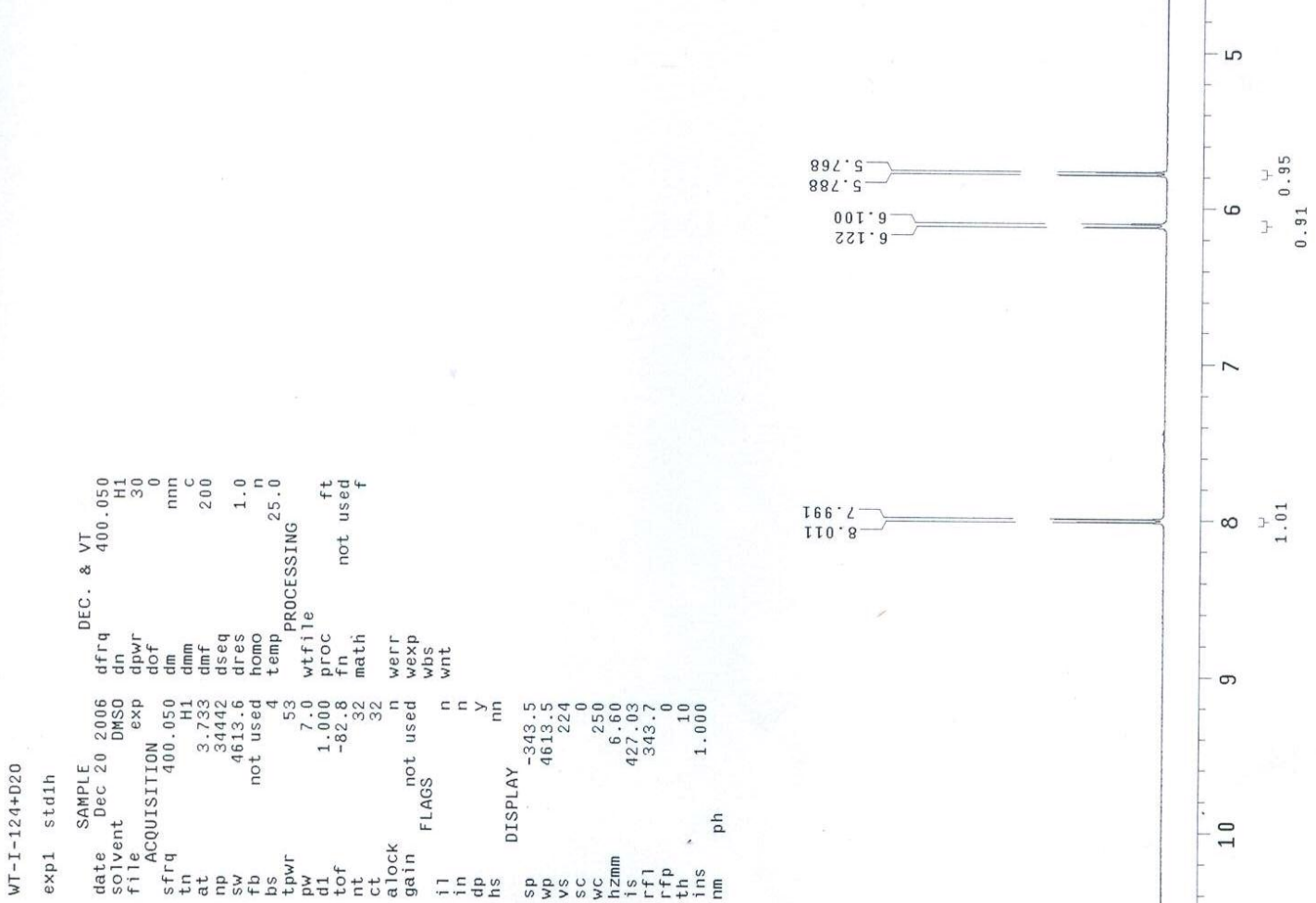

$\underset{T 10.8}{I 66.2}$

$\infty \stackrel{\overrightarrow{+}}{\overrightarrow{+}}$ 흠 ․․르 


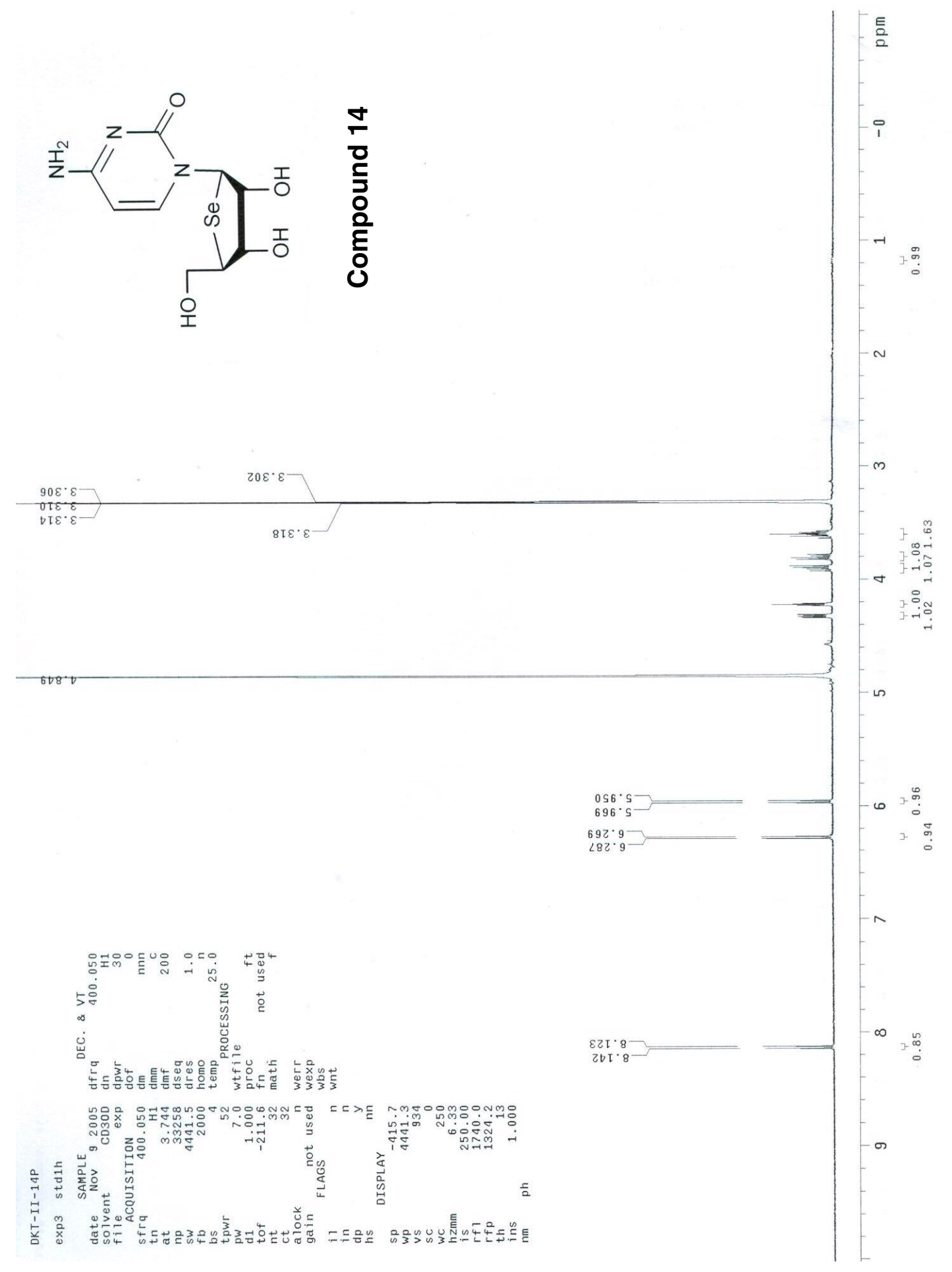



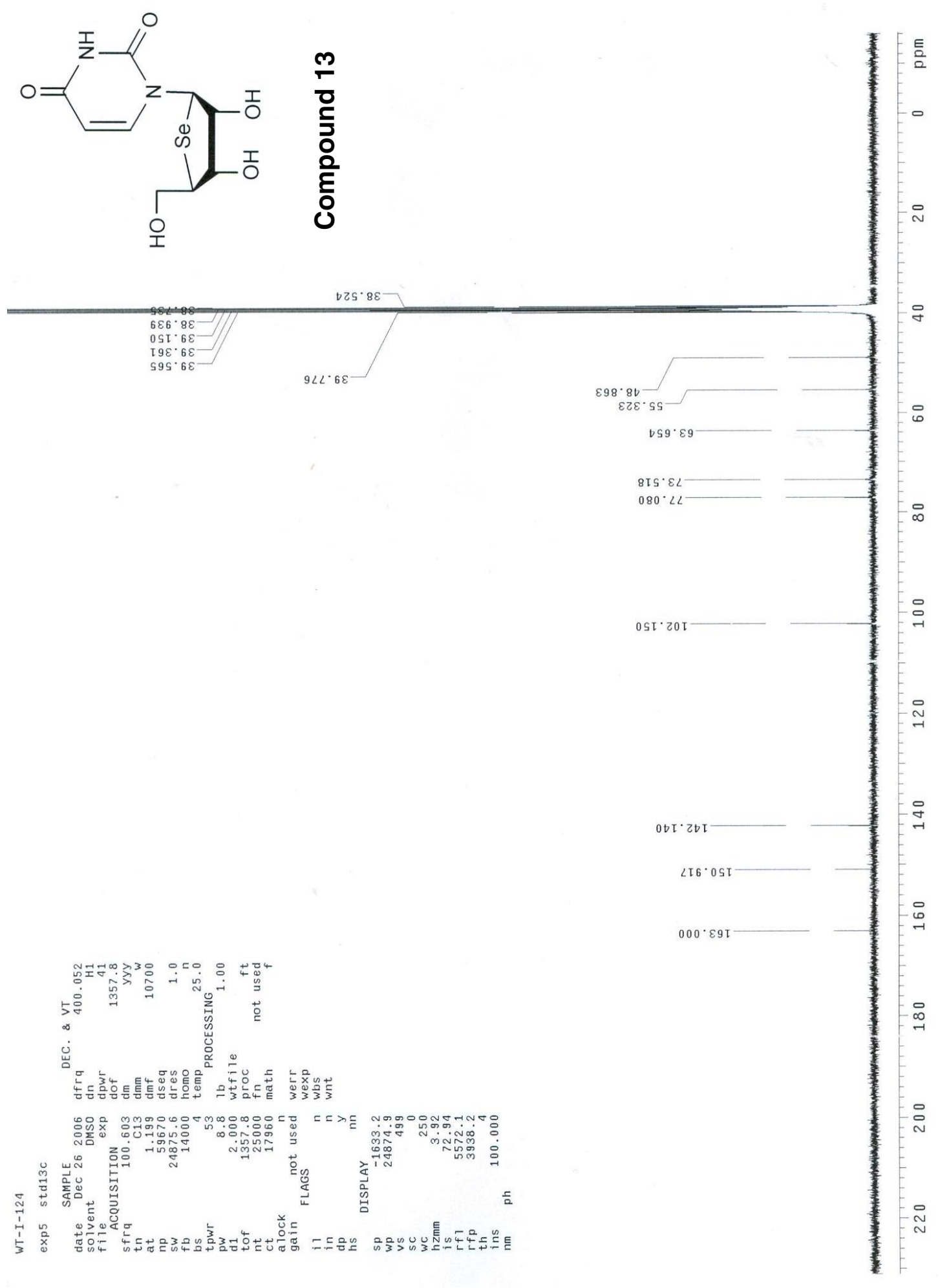


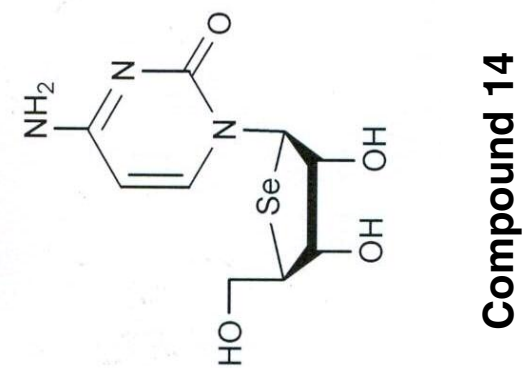

$Z$ IS. $8 b$
$b Z L \cdot 8 b$

$0 \mathrm{SI} \cdot 6 \mathrm{~b}$

$\varepsilon 9 \varepsilon \cdot 6 \mathrm{~b}$

$69 b \cdot 6 b$

$88 L \cdot 6 \mathrm{t}$

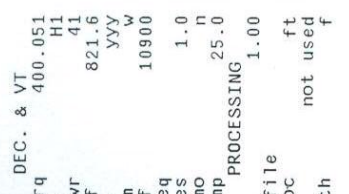

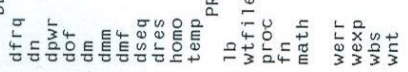

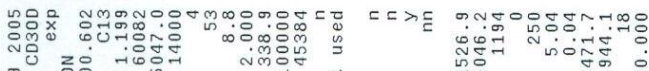

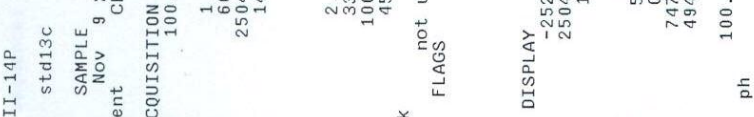

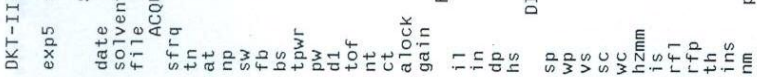

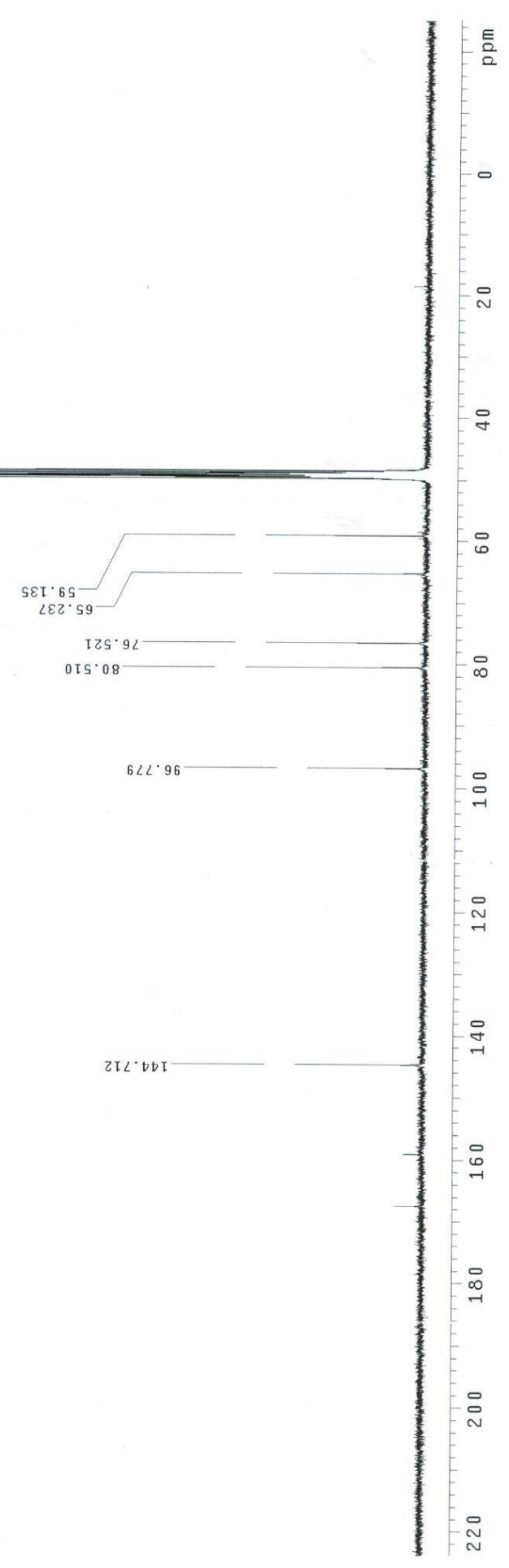

